\title{
Discriminating the Direction of Second-Order Motion at Short Stimulus Durations
}

\author{
ANDREW M. DERRINGTON,* DAVID R. BADCOCK, $\dagger$ G. BRUCE HENNING $\ddagger$
}

Received 21 October 1992; in revised form 29 January 1993

\begin{abstract}
We measured the ability of human observers to discriminate the direction of motion of different spatial patterns presented for durations ranging from 0.021 to $0.67 \mathrm{sec}$. The patterns were: (1) a vertical grating (spatial frequency $0.93 \mathrm{c} / \mathrm{deg}$ at $5 \%$ contrast); (2) a "beat" pattern made by adding vertical gratings of 6.3 and $5.4 \mathrm{c} / \mathrm{deg}$ both at $5 \%$ contrast moving in opposite directions (this pattern appears as a horizontally moving, $0.93 \mathrm{c} / \mathrm{deg}$ "beat"; i.e. spatial variation in the contrast of a stationary vertical grating of $5.8 \mathrm{c} / \mathrm{deg}$ ); and (3) a "plaid" pattern made by adding gratings of $5.9 \mathrm{c} / \mathrm{deg}$ orientated \pm 81 deg from vertical (this pattern can also be expressed as a horizontally moving $1.9 \mathrm{c} / \mathrm{deg}$ beat in a horizontal grating of $5.8 \mathrm{c} / \mathrm{deg}$ ). The direction of motion of the grating and the plaid pattern were discriminable at all durations tested. The direction of motion of the beat could only be discriminated at durations above approx. 200 msec. We suggest that this is a consequence of the fact that the moving beat is only visible to second-order mechanisms, and that second-order mechanisms for the analysis of motion operate more slowly than first-order mechanisms.
\end{abstract}

Motion Plaid Beat Low-level motion Direction-selectivity Psychophysics

\section{INTRODUCTION}

\section{Low-level and high-level motion analysis}

In principle, information about motion can be extracted from the visual image by a variety of different strategies that may or may not reflect modes of operation of visual mechanisms. However, it is now general practice to distinguish between low-level mechanisms, that calculate a motion signal by spatio-temporal correlation (or Fourier analysis) of the outputs of linear filters applied to the image (Adelson \& Bergen, 1985; Braddick, 1974; Reichardt, 1961; van Santen \& Sperling, 1984; Watson \& Ahumada, 1985), and high-level mechanisms, which require the image to be processed in some non-linear way to extract data which can then be used for a correlation analysis (Anstis, 1980; Braddick, 1980; Cavanagh \& Mather, 1989; Chubb \& Sperling, 1989; Ullman, 1979). Although the theoretical distinction between low-level and high-level mechanisms is easy to draw, it is not always easy to distinguish between their operation in experiments. In particular, although one can isolate a high-level mechanism simply by using a stimulus that requires the appropriate form of preprocessing to extract any motion signal (Cavanagh \& Mather, 1989; Chubb \&

\footnotetext{
*Department of Physiological Sciences, The Medical School, Newcastle upon Tyne NE2 4HH, England.

†Department of Psychology, University of Melbourne, Parkville, Victoria 3052, Australia.

†Department of Experimental Psychology, University of Oxford, South Parks Road, Oxford OX1 3UD, England.
}

Sperling, 1989; Derrington \& Badcock, 1985), isolating a low-level mechanism is more difficult, because, in general, any motion signal that is available to a low-level mechanism is also available to high-level mechanisms.

\section{Decreasing duration favours "low-level" mechanisms}

One stimulus manipulation that does appear to favour a low-level mechanism over a high-level mechanism is to reduce the duration (or to raise the temporal frequency) of the stimulus. The effect has been widely studied in a variety of ambiguous stimuli in which low-level and high-level systems signal different motions. Anstis (1970) showed that the motion of a grating that alternated periodically between two different orientations could be perceived in two ways: with long intervals between the alternations, the motion was seen as global rotation of the grating, a "high-level" percept because in order to perceive global rotation, one must first extract the form of the grating. With shorter intervals between the position changes, the motion was seen as a local change at the spatial intersections within the two gratings. This percept was presumably mediated by a low-level motion system, since it would be produced by a spatio-temporal correlation of the local illuminance values of the retinal image. Similar changes from "high-level" to "low-level" motion percepts have been reported as a consequence of reducing the interstimulus interval in apparent motion displays that could be interpreted either as motion of individual local elements of the pattern (low-level) or as a coordinated movement of the whole pattern (highlevel) (Braddick, 1980; Petersik \& Pantle, 1979). 
A simpler situation results when low-level and highlevel mechanisms signal opposite directions of motion (Chubb \& Sperling, 1989; Derrington, Badcock \& Holroyd, 1992). In such cases it has been shown that high-level mechanisms tend to dominate at low spatial and temporal frequencies (Derrington et al., 1992). However, the interpretation of results where the two mechanisms signal opposite directions of motion is a

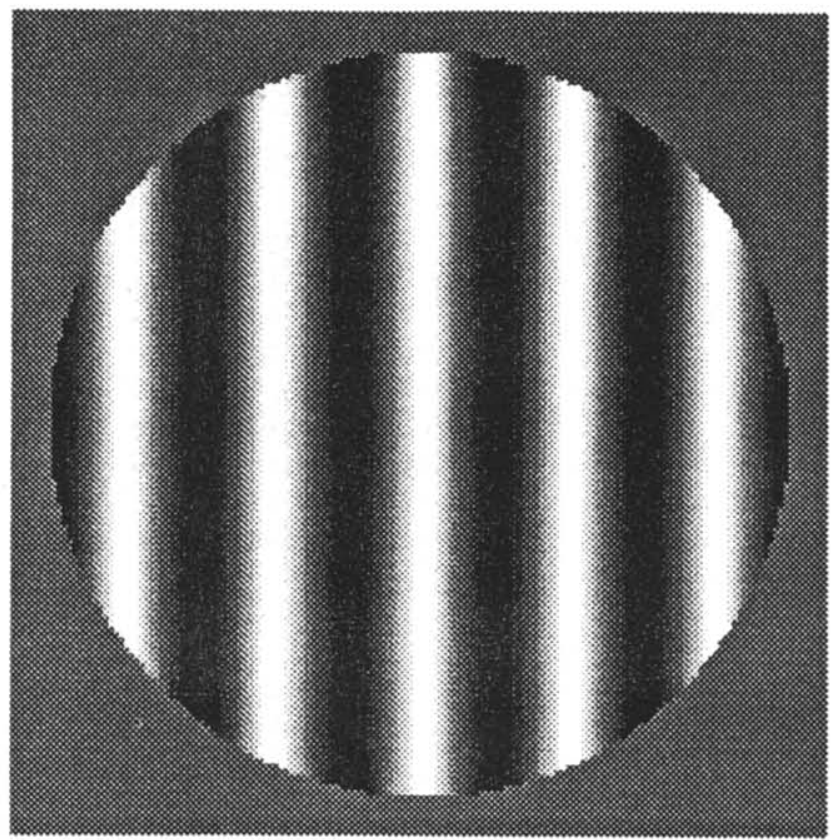

C

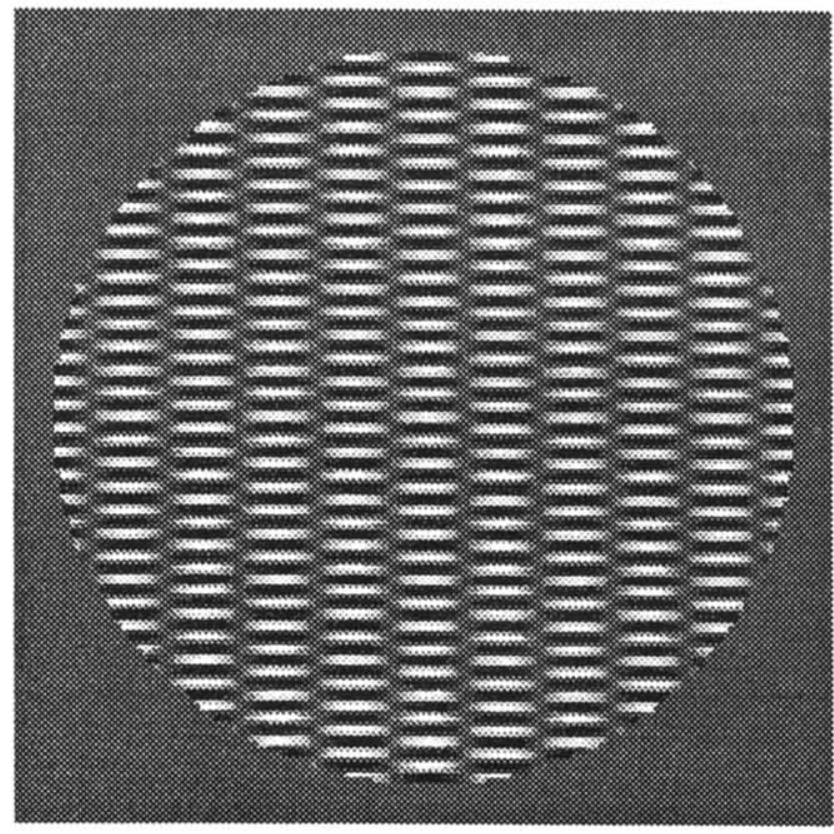

b

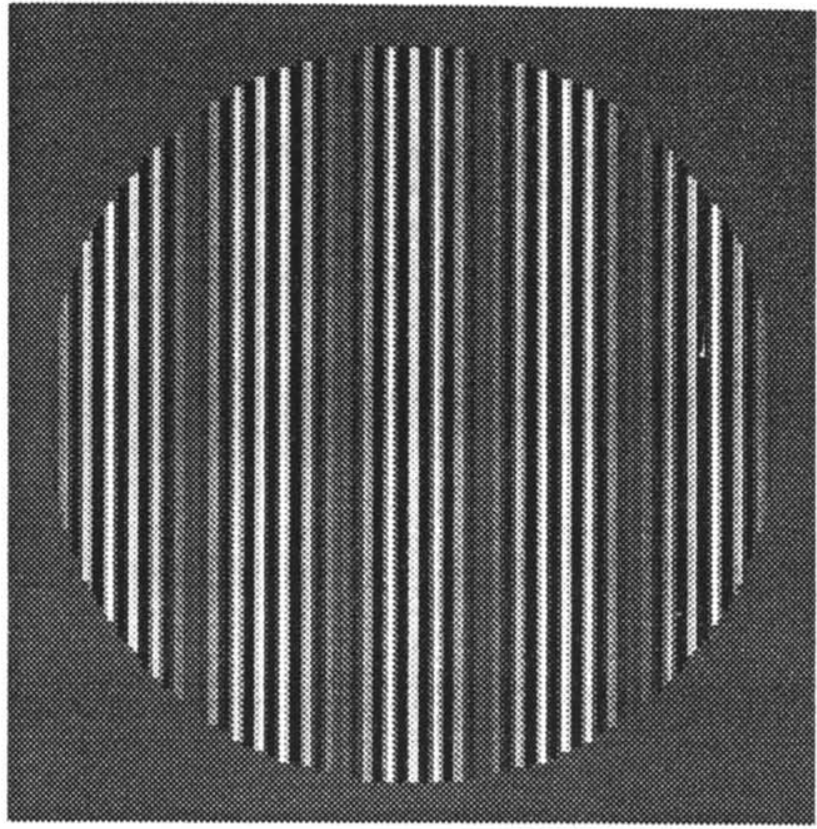

d

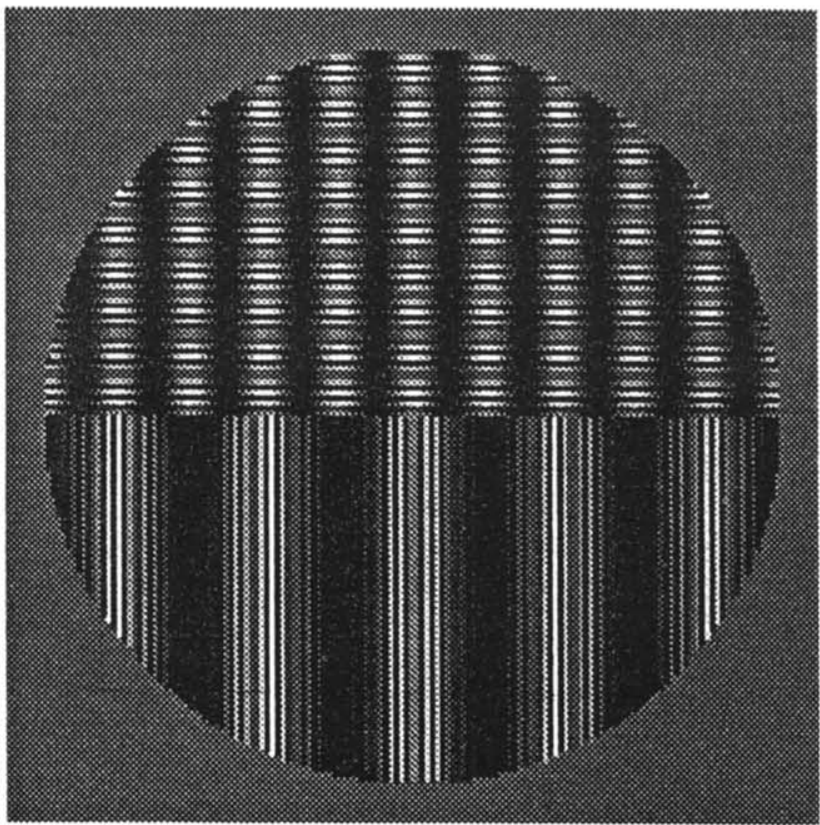

FIGURE 1. First-order and second-order stimuli used in this study; each circular disk shows a high-contrast representation of one of the patterns. If the figure is viewed so that the diameter of the disk subtends 5 deg the pattern will be approximately the size used, although the spatial frequencies will not be exactly the same. (a) Sinusoidal grating, spatial frequency approx. $1 \mathrm{c} / \mathrm{deg}$. (b) Beat pattern of period $1 \mathrm{deg}$, made by adding two vertical sinusoidal gratings of 5.8 and $6.8 \mathrm{c} / \mathrm{deg}$. The pattern appears as a low-frequency periodic spatial variation in the contrast of a vertical grating of $6.4 \mathrm{c} / \mathrm{deg}$. (c) Plaid pattern made by adding two sinusoidal gratings of $6.4 \mathrm{c} / \mathrm{deg}$, orientated $\pm 81 \mathrm{deg}$ from vertical. The pattern appears as a vertically orientated periodic variation in the contrast of a horizontal grating of high spatial frequency. (d) Patterns made by squaring and normalizing (b) and (c) to reveal the spatial patterns that may be available for second-order motion analysis. The upper half of the disk shows the normalized square of (c) - the plaid pattern-which has a horizontal period of $0.5 \mathrm{deg}$. The lower half of the disk shows the normalized square of (b) - the beat pattern-which has a horizontal period of approx. 1 deg. 


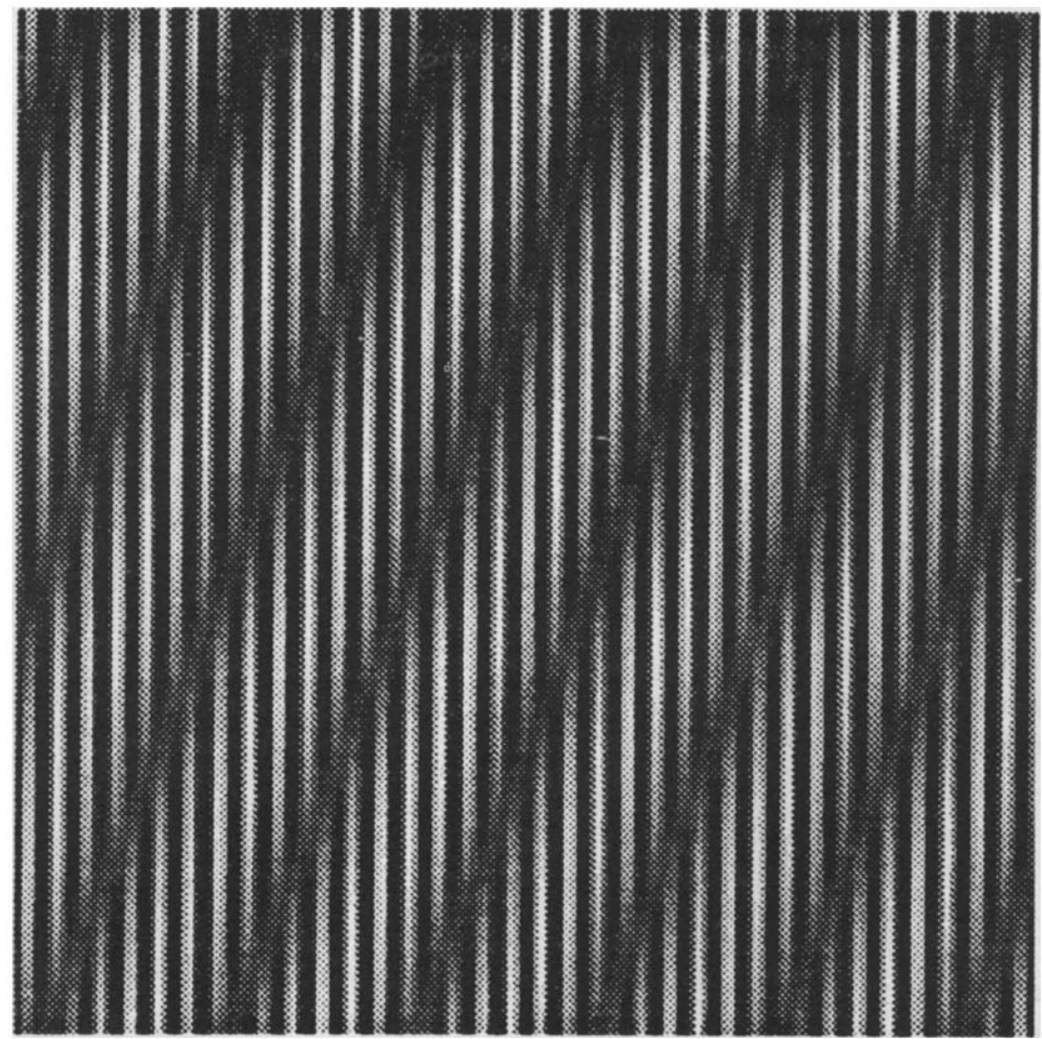

FIGURE 2. Time-space density plot of a moving beat. The variation in density along the horizontal axis represents the distribution of luminance as a function of horizontal distance across the display; the vertical variation shows how this distribution changes with time. The motion of the beat is shown as a rightward displacement of the contrast modulation as one moves up the plot. Note that the high frequency grating is stationary, as indicated by the fact that its bars are vertical, i.e. constant over time.

limited by the fact that changes in perceived direction of motion could result from changes either in the low-level mechanism or in the high-level mechanism, thus it is not clear what the limits of the high-level mechanism are. Nevertheless, it is generally assumed that one can isolate the low-level motion system simply by using brief presentations. For example Yo and Wilson (1992) suggest that the way in which the perceived axis of motion of certain plaid patterns changes with the duration of presentation can be explained by a model in which the signals from second-order and first-order motion analyses are combined (Wilson, Ferrera \& Yo, 1992).

Yo and Wilson (1992) assume that perceived axis of motion changes with duration because the second-order analysis is assumed to take longer than the first-order analysis (Yo \& Wilson, 1992). Thus in brief stimuli only first-order mechanisms are able to provide signals, but as the stimulus duration is increased, signals from the slower second-order mechanisms gradually become available and influence the motion percept.

The assumption that second-order mechanisms operate more slowly than first-order mechanisms has never been directly tested. The main aim of this paper is to test this assumption, and to clarify the nature of the temporal limitations on second-order motion mechanisms. To that end we have chosen to investigate the effects of exposure-duration on the detectability of the motion of a very simple second-order stimulus, a "beat" pattern, which is formed by adding together two gratings of about six c/deg which differ in frequency by about one $\mathrm{c} / \mathrm{deg}$. The pattern appears as a spatially periodic variation in the contrast of a grating whose frequency is the mean of the frequencies of the two components [see Fig. 1(b)]. The period of the variation in contrast is equal to the difference between the frequencies of the two components; and, if they are made to move in opposite directions with equal temporal frequencies, the low-frequency contrast variation moves but the high frequency "carrier" grating remains stationary (Derrington \& Badcock, 1985) (see Fig. 2). This stimulus is second-order in the sense that spatio-temporal filtering of the raw luminance values would not reveal a moving beat pattern. Spatio-temporal filtering could, of course, be used to reveal the motion of the components of the beat, which, as stated, move in opposite directions with equal temporal frequency. The same analysis applied to the square of the luminance values would reveal the moving beat, as is shown in Fig. 1(d) (Derrington \& Badcock, 1985).

Figure 1(d) shows patterns produced by squaring either a beat (lower half) or a plaid (upper half). In both cases the squaring introduces a clear spatial modulation of luminance which follows the spatial modulation of contrast in the original pattern. Those parts where contrast is high appear bright, and those parts where contrast is low appear dim. When the spatial variation in contrast is made to move by moving the beat or the plaid, the luminance modulation introduced by squaring also moves, and its motion would be detectable by 
spatio-temporal filtering or correlation occurring after the non-linearity. Thus appropriate filtering would be able to extract a second-order motion signal from signals generated by non-linear receptive field mechanisms (Derrington, 1987, 1990).

\section{METHODS}

\section{Stimuli}

Patterns were generated using a RGB framestore that was part of a purpose built display controller, the Cambridge Research Systems VSG 2/1 (Derrington \& Suero, 1991), and displayed on a Joyce Electronics monitor with a P4 (bluish white) phosphor and highbandwidth (non-linear) $\mathrm{Z}$ amplifier. Details of the procedures used to avoid distortion are given below. The three DAC outputs of the framestore were summed with different gains to give more precise control of contrast (Pelli \& Zhang, 1991). On each frame of the display (frame frequency $180 \mathrm{~Hz}$ ) a moving sinusoidal grating was presented within a circular patch, the diameter of which subtended $5 \mathrm{deg}$ at the $2-\mathrm{m}$ viewing distance. The mean luminance of the display was $47 \mathrm{~cd} \cdot \mathrm{m}^{-2}$; the illuminated area subtended $7.7 \mathrm{deg}$ horizontally by $6.4 \mathrm{deg}$ vertically, and it had a dark surround. The room was dimly illuminated.

Normally two different patterns were interleaved, each member of the pair being presented on alternate frames. Two different stimulus pairings were used: a vertical grating paired with a blank field; and two spatiallysuperimposed gratings of equal contrast but different spatial frequencies, to produce a "beat" pattern, illustrated in Fig. 2(b), or of different orientations, to produce a "plaid" pattern (Adelson \& Movshon, 1982). The grating pattern is described by the equation

$$
L(x, t)=L_{\mathrm{m}}\{1+C \cos [2 \pi(f x+g t)+\phi]\}
$$

where $L_{\mathrm{m}}$ is the mean luminance, $C$ is the contrast, $f$ is the spatial frequency, $g$ is the temporal frequency and $\phi$ is a phase term. The beat or plaid pattern was made by adding together two sinusoidal gratings of different spatial frequencies or orientations, and is described by the general equation

$$
\begin{aligned}
L(x, y, t)= & L_{\mathrm{m}}\left\{1+C \cos \left[2 \pi\left(u_{1} x+v_{1} y+g_{1} t\right)+\phi\right]\right. \\
& \left.+C \cos \left[2 \pi\left(u_{2} x-v_{2} y+g_{2} t\right)+\phi\right]\right\} .
\end{aligned}
$$

In the case of the beat pattern, $v_{1}=v_{2}=0, u_{1}=\left(f_{\mathrm{c}}+f_{\mathrm{e}}\right)$, $u_{2}=\left(f_{\mathrm{c}}-f_{\mathrm{e}}\right)$ and $g_{1}=-g_{2}=g$. In the case of the plaid pattern, $u_{1}=u_{2}=u, v_{1}=v=-v_{2}$ and $g_{1}=g_{2}=g$, so the two components were orientated symmetrically $\pm \arctan (v / u)$ from the vertical, and their spatial frequency was $\sqrt{ }\left(u^{2}+v^{2}\right)$.

In the case of the beat pattern equation (2) can be rewritten as

$$
\begin{aligned}
L(x)=L_{\mathrm{m}}\left[1+2 C \cos \left(2 \pi f_{\mathrm{e}} x+\right.\right. & \left.\pi g t+\phi_{\mathrm{c}}\right) \\
& \left.\times \cos \left(2 \pi f_{\mathrm{c}} x+\phi_{\mathrm{c}}\right)\right]
\end{aligned}
$$

expressing the pattern as the product of a moving cosinusoidal envelope, of spatial frequency $f_{\mathrm{e}}$ and temporal frequency $g / 2$, and a static cosinusoidal carrier of spatial frequency $f_{\mathrm{c}}$. However the spatial modulation in the contrast of the carrier has a periodicity twice that of its envelope [see Fig. 1(b)], and so we refer to the spatial frequency of the beat as $f_{\mathrm{b}}$, where $f_{\mathrm{b}}=2 f_{\mathrm{e}}$, and to its temporal frequency as $g$.

The following precautions were taken to ensure that the spatial waveforms of the patterns were not significantly distorted by the non-linear relationship between $\mathrm{Z}$ amplifier input voltage and screen luminance. First, the luminance of a small patch of display was measured at 16-20 different voltages chosen to generate luminances approximately equally spaced throughout the available range. Then the function $L=a V^{b} /(V+c)^{b}$ was fit to the measured values by using a general purpose minimization routine (Chandler, 1965) to estimate the values of $a, b$ and $c$ which produced the lowest root mean square error (typically below $1 \mathrm{~cd} \cdot \mathrm{m}^{-2}$ ). The inverse function was then used to calculate lookup tables to generate sinusoidal gratings free from distortion; we have referred to this process as gamma correction. Finally, to remove the possibility that any error in this procedure might generate a difference-frequency distortion product, that could be used by observers to detect the motion of beat or plaid patterns (Derrington \& Suero, 1991; Henning, Hertz \& Broadbent, 1975) the two components of a beat pattern were always displayed on different frames, so that cross-products (and the associated difference-frequency distortion products) could not be generated in the display.

All patterns were modulations of luminance without changes in chromaticity, and were presented with abrupt onset and offset. The spatial frequency of the grating was $0.93 \mathrm{c} / \mathrm{deg}$; the components of the plaid pattern were $6.0 \mathrm{c} / \mathrm{deg}$ : orientated $\pm 81 \mathrm{deg}$ from vertical; the spatial frequencies of the components of the beat pattern were 5.4 and $6.3 \mathrm{c} / \mathrm{deg}$ which gave a spatial frequency of 0.93 $\mathrm{c} / \mathrm{deg}$ for the beat pattern. The plaid pattern had a horizontal period of $1.1 \mathrm{deg}$, but an apparent horizontal period of $0.54 \mathrm{deg}$, half that of the beat pattern. In preliminary experiments, we found that raising the beat frequency or increasing the angle between the components of the plaid pattern caused a tendency for the moving patterns to break up.

The sinusoidal grating patterns were generated by storing lookup-table index values in the parts of display memory that were displayed as the circular patch on alternate frames. The memory locations corresponding to the rest of the visible screen area contained the index of the lookup table entry containing the mean luminance. Separate lookup tables, each containing 251 gamma-corrected luminance values corresponding to a full cycle of a sine-wave of contrast 0.1 , were maintained for each pattern. Thus the part of display memory representing each pixel contained a number which indicated the phase of the sinusoid at that point in the picture. The lookup table was used to convert that phase into the three numbers which, when loaded in the 8-bit DACs, gave the luminance required at that phase for a 
sinusoidal grating of contrast 0.1 . Because each grating was interleaved either with another, or with a blank field, the time-average contrast of each grating was always 0.05 .

Each pattern could be made to move smoothly within its circular patch by loading a new lookup table each time the pattern was displayed ( 90 times per sec). The smallest unit of phase shift was $1 / 251$ cycles, giving a temporal frequency resolution of $0.36 \mathrm{~Hz}$ independent of the spatial frequency of the pattern. To obtain finer resolution of temporal frequency at the expense of smoothness of movement, phase shifts were made in units of $1 / 2008$ cycles (1/8 waveform samples) and rounded down to the nearest whole step on each frame. Thus the slowest possible motion, $0.045 \mathrm{~Hz}$, would result in a phase shift once every 8 frames. The lack of smoothness in the motion was not noticeable.

The shape of the spatial and temporal windows within which the patterns were displayed deserves some comment. In both cases the onset and offset was sharp, rather than being smoothed with a Gaussian profile. In the case of the spatial window the effect is likely to be small, as the size of the patch was large with respect to the period of the patterns involved. The same cannot be said of the temporal window, and it should be borne in mind that changing the shape of the temporal window would produce substantial changes in the shape of the temporal frequency spectrum of the pattern at frequencies close to its nominal temporal frequency. However, in the work reported here we use a rectangular window for three reasons:-first, it allows us to specify the duration of the stimulus unequivocally, second, it allows direct comparison with the work of Yo and Wilson (1992), and finally we have obtained results qualitatively similar to the main one reported here using both Gaussian (Derrington \& Henning, unpublished results) and raised cosine (Cropper \& Derrington, 1993) temporal envelopes.

\section{Subjects}

The observers were one of the authors, and a wellpractised paid observer who knew nothing of the theoretical background of the experiments. They viewed the screen without head restraint, and with natural pupils and accommodation. They were given a fixation mark, and were instructed to fixate. They wore their prescribed spectacle corrections.

\section{Procedures}

A temporal two-alternative forced-choice (2-AFC) paradigm was used in conjunction with the method of constant stimuli to obtain psychometric functions ( 50 observations per point) relating performance in direction-discrimination tasks to stimulus duration. Each trial was initiated by a key-press, and consisted of two temporal intervals signalled to the observer by bursts of audible noise. During one interval, chosen at random, a pattern was presented moving to the left, during the other interval the same pattern was presented moving to the right. The observer's task was to signal, by pressing

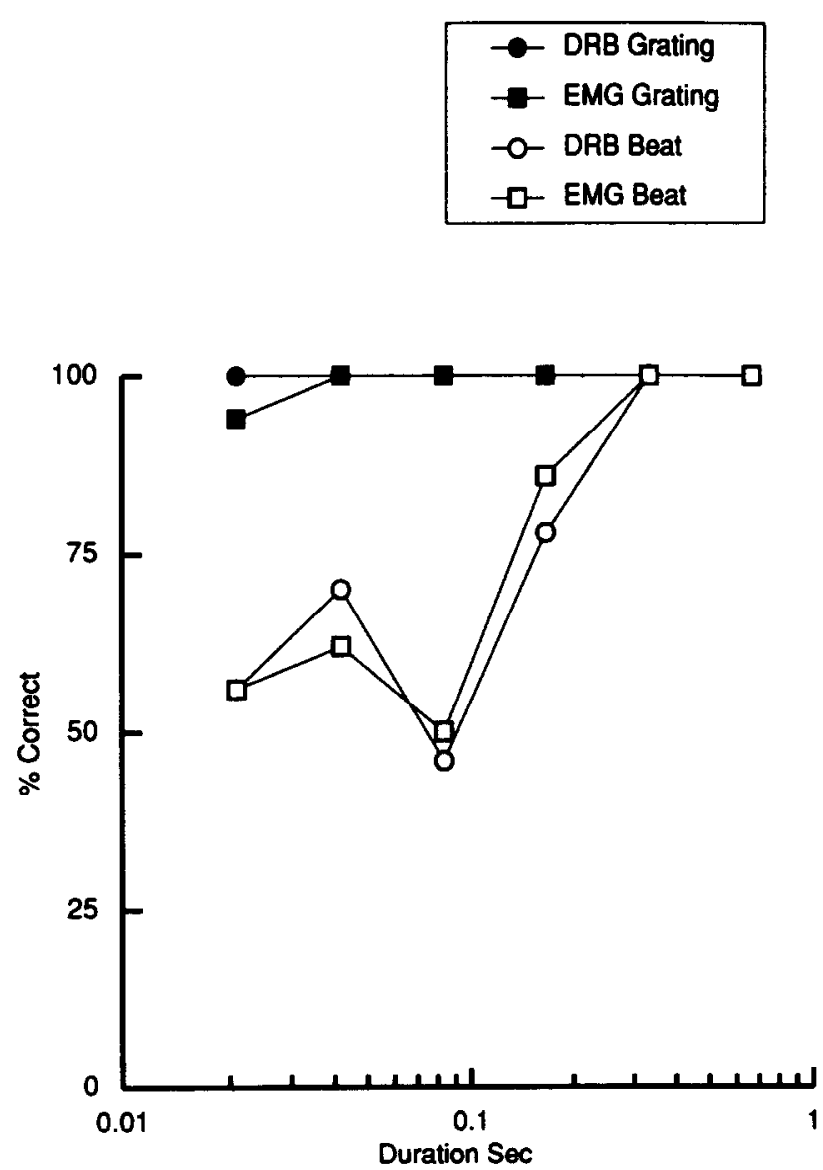

FIGURE 3. Performance as a function of duration in discriminating the direction of motion of a grating of spatial frequency $0.93 \mathrm{c} / \mathrm{deg}$ $(\square)$ ) and a beat pattern $(\square \bigcirc)$ made by adding together gratings of spatial frequency 5.39 and $6.32 \mathrm{c} / \mathrm{deg}$. Velocity was changed with duration so that each pattern moved smoothly through one-quarter of its horizontal spatial period during each observation interval. Contrast of the grating and of the components of the beat pattern was 0.05 . Each point is based on 50 observations from observers DRB and EMG.

a key-switch, the interval in which the pattern had moved to the left. Observers were given no feedback as to the correctness of their responses on individual trials.

The stimulus to be presented on each trial was selected at random from the set of six used for the current block of trials; with the constraint that no stimulus as presented for the $n$th time until all stimuli had been presented $n-1$ times. A computer (Tandon PCA 20), containing the VSG2/1, controlled the selection, generation and display of stimuli, and the recording of responses.

\section{RESULTS AND DISCUSSION}

\section{Experiment 1: comparison of beat and grating}

Figure 3 shows the performance of two observers discriminating the direction of motion of a first-order pattern (a sinusoidal grating) and a second-order pattern (a beat) of the same spatial period. In both cases performance is plotted as a function of the stimulus duration. The speed of the moving pattern covaried with 


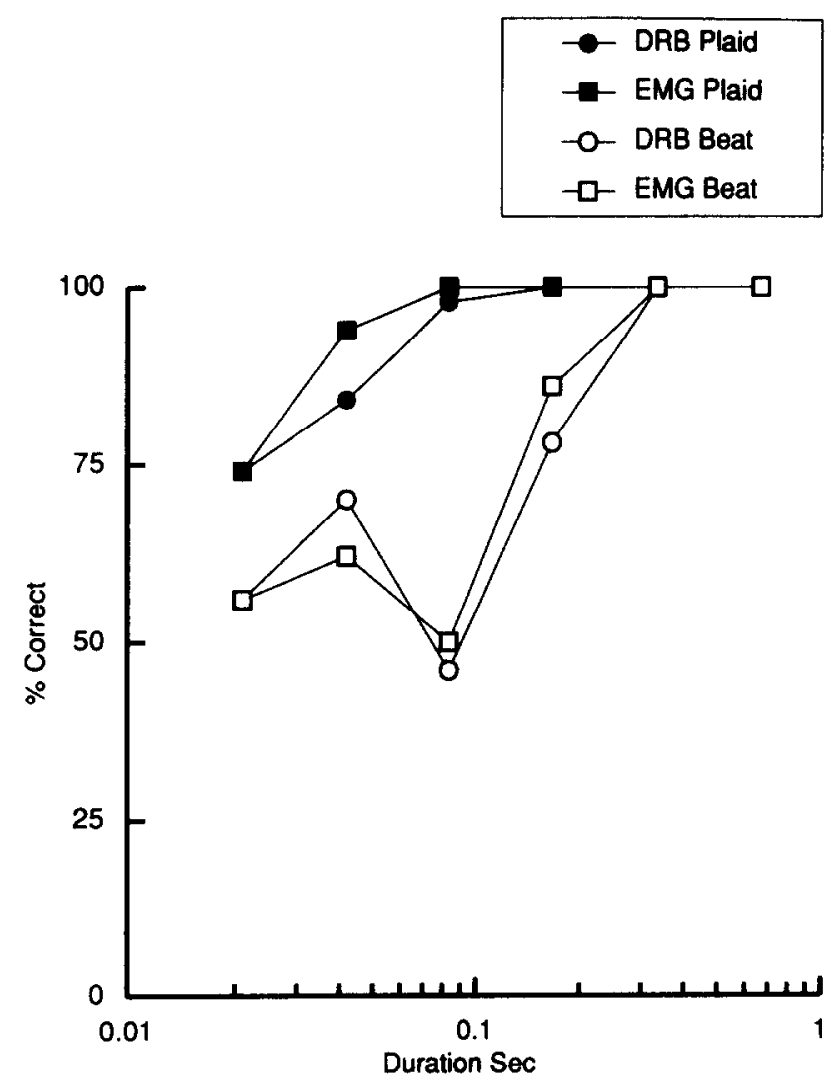

FIGURE 4. Performance of the two observers as a function of duration in discriminating the direction of motion of a plaid pattern made by adding two gratings of spatial frequency $5.95 \mathrm{c} / \mathrm{deg}$ and contrast 0.05 , oriented $\pm 81 \mathrm{deg}$ from vertical $(\square \bigcirc)$. Open symbols show performance with beat pattern replotted from Fig. 3. Other details are as for Fig. 3.

the duration, so that the pattern always moved through one-quarter of its spatial period during each presentation. There is a marked difference between the results obtained with the two patterns. With the sinusoidal grating performance is essentially perfect at all durations from $0.021 \mathrm{sec}$, whereas the motion of the beat cannot be discriminated at short durations; performance reaches $75 \%$ correct at a duration of about $200 \mathrm{msec}$. In order for the observers to see the motion of the beat it must be presented for at least ten times as long as is required to see motion of the grating. This result suggests that the second-order mechanism which signals the motion of the beat is in some sense more sluggish than the mechanism which signals the motion of the grating.

\section{Experiment 2: comparison of beat and plaid}

It is possible that the difference between the levels of performance obtained with the two patterns is a consequence of the fact that the components of the beat pattern are higher in spatial frequency. To test this possibility we measured performance with a plaid pattern with components of similar spatial frequency to those in the beat pattern. The comparison is shown in Fig. 4, where direction-discrimination performance is plotted as a function of duration; the data obtained using the beat pattern are replotted for comparison. Although performance with the plaid is not quite as good as performance with a low-frequency grating, performance is substantially better than that obtained with the beat except at durations above $200 \mathrm{msec}$ (where performance is errorless). In facl, at the slowest duration, performance is approx. $75 \%$ correct, indicating that the mechanism which processes the motion of the plaid is about 10 times faster than that which processes the beat.

The esssential difference between the plaid and the beat pattern is that, in principle, it is possible to analyse the motion of the plaid pattern by linear mechanisms, either by a two-stage process in which the pattern's two-dimensional velocity vector is calculated from the signals provided by the one-dimensional analysers which signal the motion of the gratings (Adelson \& Movshon, 1982), or directly by analysing the motion of local segments of the (very short) vertical edges in the pattern (Derrington \& Badcock, 1992). Whatever the first-order process is, it is clearly much faster, in the sense that it can detect motion in a much briefer stimulus, than the second-order process which must be used to analyse the motion of the beat.

\section{Experiment 3: systematic exploration of effects of varying duration and temporal frequency}

In Expts 1 and 2 speed was adjusted so that the pattern moved through one-quarter of its apparent spatial period during the observation interval, with the consequence that its speed (or temporal frequency) was inversely related to its duration. At the shortest duration, the nominal temporal frequency was approx. $12 \mathrm{~Hz}$ (although the brief duration will have ensured that the stimulus really contained a broad band of temporal frequencies centred on this value). Thus there is a possibility that the decline in direction-of-motion discrimination performance at short durations could be a consequence of the increase in temporal frequency rather than the reduction in duration. To test this we studied performance as a function of stimulus duration for a number of fixed temporal frequencies using the same three stimuli and the same two observers. The results are shown in Fig. 5.

Figure $5(\mathrm{a}, \mathrm{b})$ shows performance in discriminating direction of motion of the sinusoidal grating as a function of the stimulus duration, with temporal frequency as a parameter. Within the range of temporal frequencies studied $(0.5-8 \mathrm{~Hz})$ performance improves with both duration and temporal frequency. Observer EMG achieves perfect performance for all speeds at a duration $<100 \mathrm{msec}$. Observer DRB is slightly worse, and there is a slight indication that he has a systematic tendency to respond incorrectly at the shortest duration. Such a tendency has been reported before (Morgan \& Cleary, 1992); and probably reflects an effect of the broadening of the temporal frequency spectrum of the stimulus at short durations. Figure 5(c,d) shows performance with the plaid pattern. The same gradual improvement with both speed and duration is seen. For the fastest moving stimuli, performance is essentially 
perfect at a duration of approx. $0.02 \mathrm{sec}$, and even for the slowest moving stimuli it is above threshold by $0.08 \mathrm{sec}$.

The data for the beat, shown in Fig. 5(e,f) are completely different. First, performance remains at chance up to a stimulus duration of $0.1 \mathrm{sec}$. Then, at longer durations, it only improves for the two lowest temporal frequencies. For the two highest temporal frequencies it actually declines to zero: the motion seen is in the opposite direction to the motion of the beat. This reversal is seen more clearly in Fig. $6(a, b)$ in which the data from Fig. 5(e,f) are replotted as functions of temporal frequency with duration as the parameter. At the shortest duration, performance is at chance for all temporal frequencies. Then, for the higher temporal frequencies, as duration increases we first see a decline in performance below chance (indicating a reversed motion percept). Finally, at the longest durations, we find that the observers are able to discriminate the direction of beat motion perfectly at low temporal frequencies, but see reversed motion at high temporal frequencies.

This reversal of the motion percept at high temporal frequencies is probably due to the fact that the beat stimulus contains two gratings of slightly different
EMG

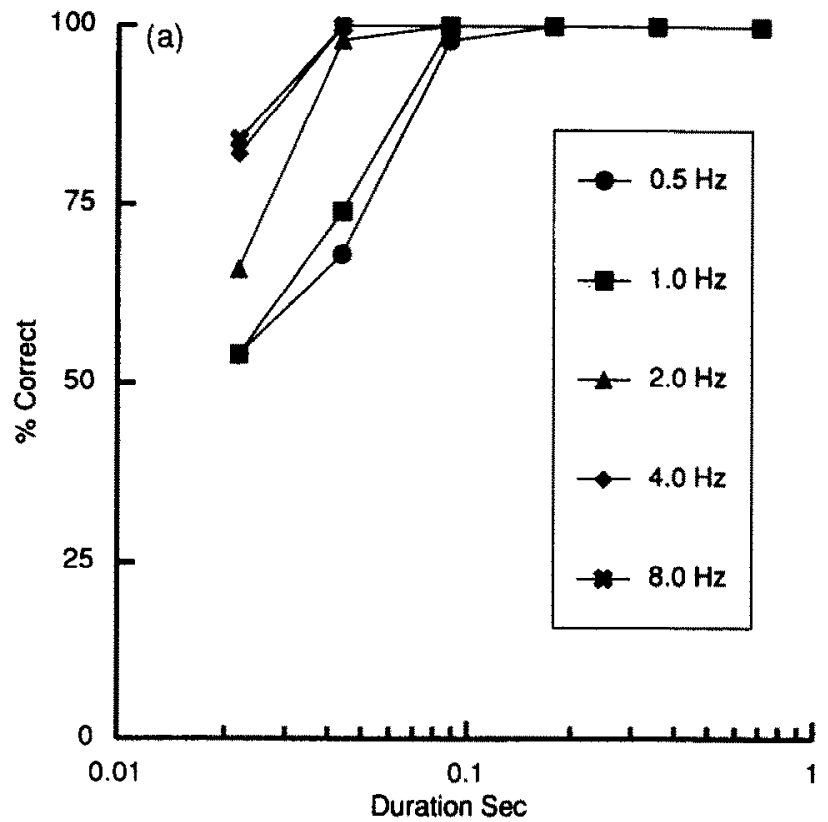

EMG

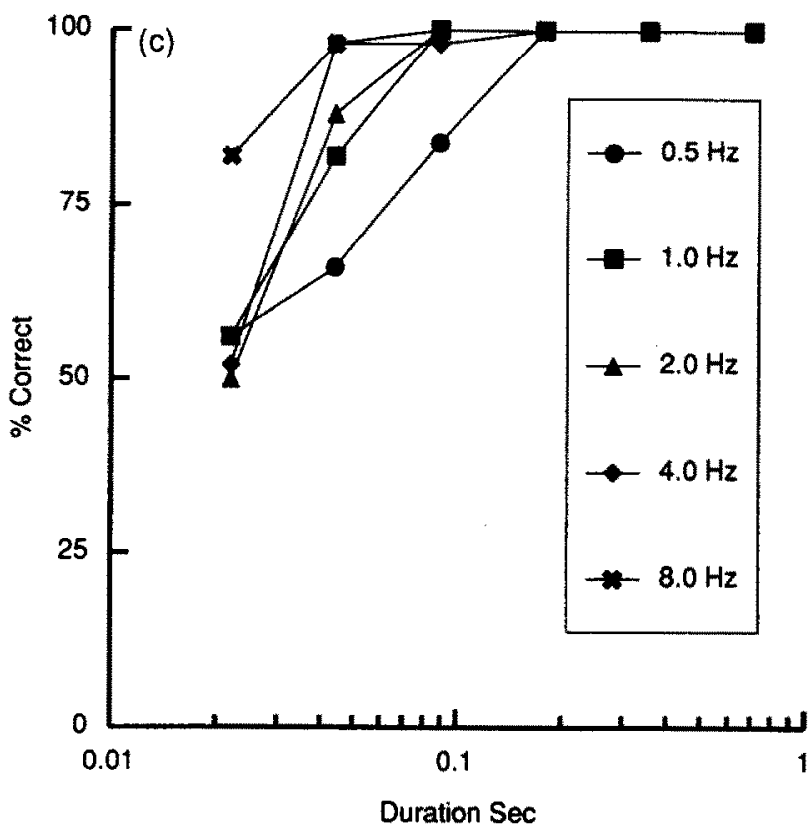

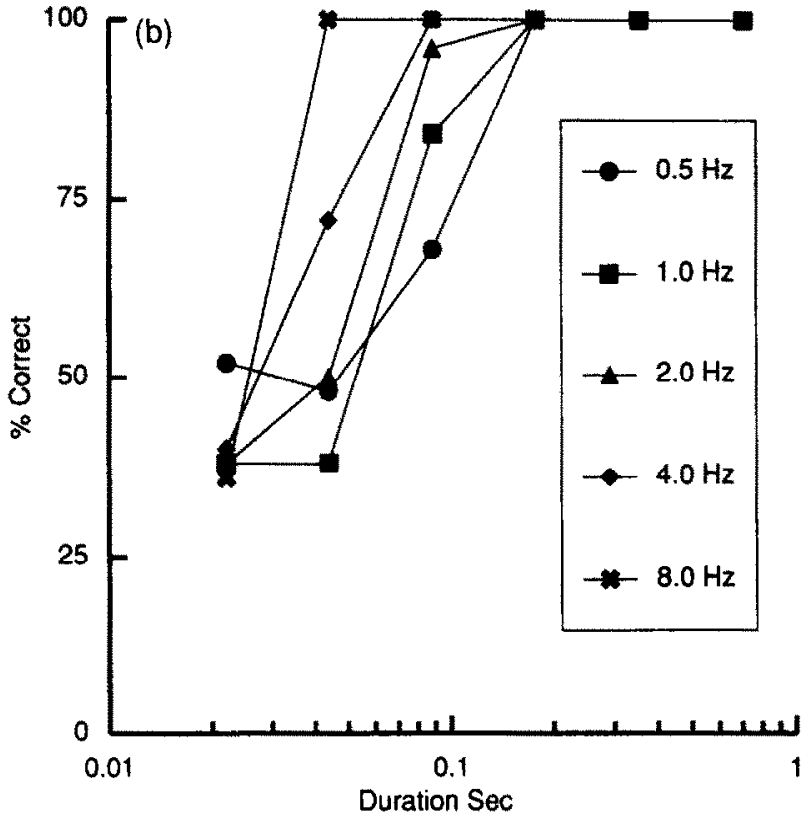

DRB

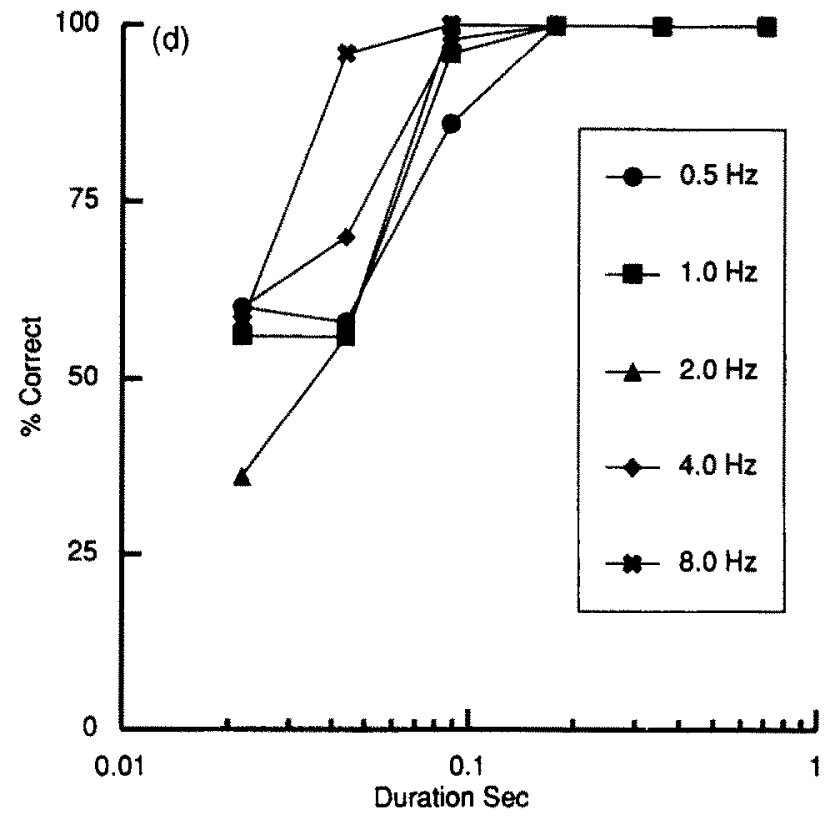

FIGURE 5(a-d). Caption overleaf. 

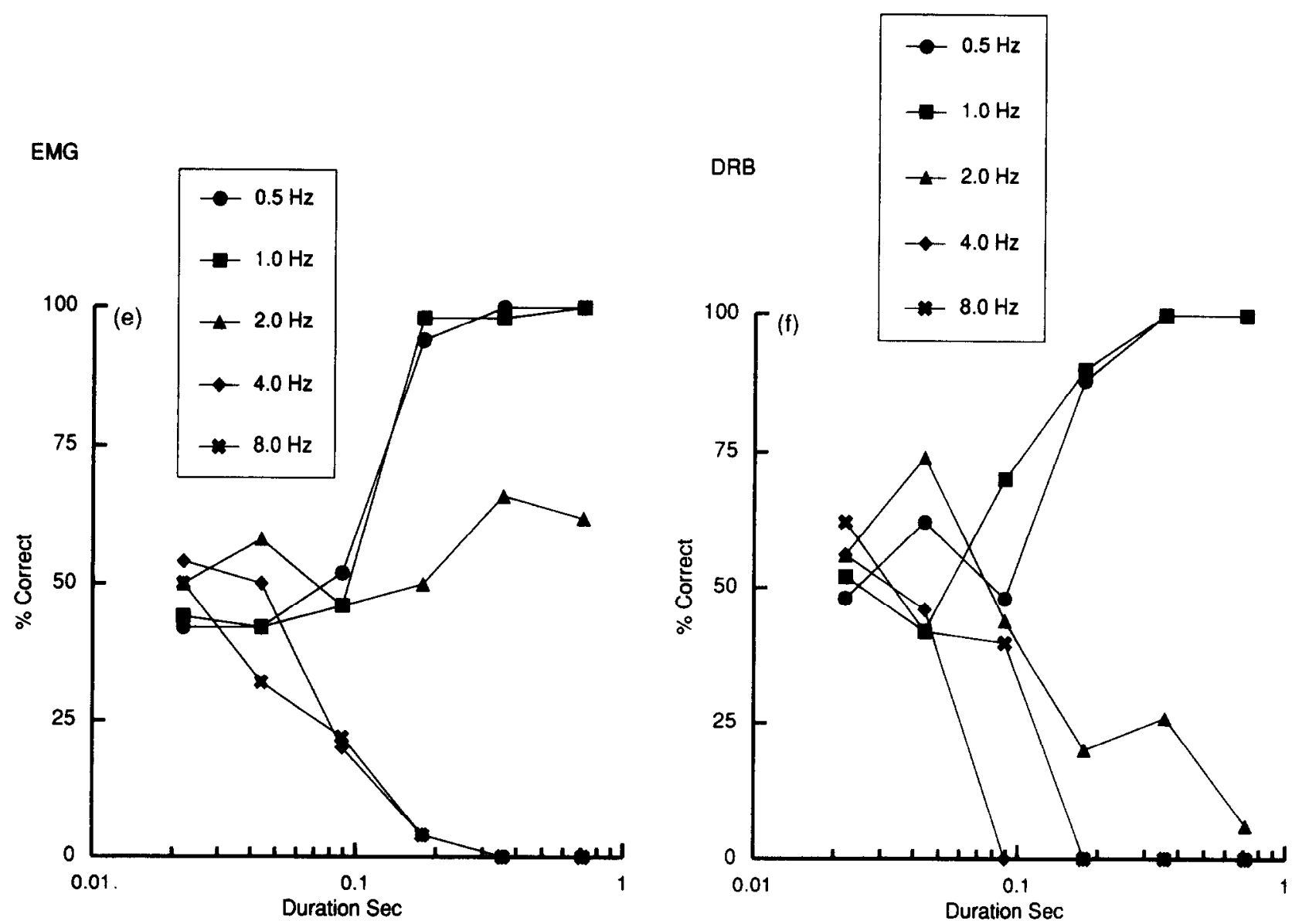

FIGURE 5. Performance of two observers discriminating the direction of motion of different patterns moving at temporal frequencies from 0.5 to $8 \mathrm{~Hz}$. Performance is plotted as a function of duration, with temporal frequency as a parameter. Other details are as in Figs 3 and 4. The stimuli used were as follows: (a, b) the grating of $0.93 \mathrm{c} / \mathrm{deg}$; (c, d) the plaid pattern; (e, f) the beat pattern.

spatial frequency moving in opposite directions at the same temporal frequency. Although this arrangement almost completely cancels the net motion energy in the stimulus, the visual system is slightly more sensitive to the component with the lower spatial frequency particularly at high temporal frequencies (Henning, 1988; Robson, 1966). The beat moves in the direction opposite that in which the lower spatial frequency component moves (Derrington \& Badcock, 1985) so it seems likely that the observers are simply responding to the motion of the most visible component of the beat. The fact that the percept of reversed motion seems to begin at shorter durations than the correct motion sensation seen at lower temporal frequencies and to increase gradually with increasing duration is consistent with this suggestion that the reversed motion percept is caused by the failure completely to balance the first-order motion signals.

\section{GENERAL DISCUSSION}

The results clearly support the conclusion that the second-order visual mechanisms which analyse the motion of beat patterns are more sluggish than those that analyse the motion of sinusoidal gratings, and that stimulus durations below about $100 \mathrm{msec}$ effectively isolate a low-level motion system which is sensitive only to first-order signals. Regardless of temporal frequency, direction-discrimination performance for beats never exceeded threshold at durations less than about $200 \mathrm{msec}$. On the other hand, performance with a plaid pattern, which has similar spatial structure to the beat pattern, but differs from it in that its motion can be analysed by first-order mechanisms, reaches threshold at a duration of approx. $20 \mathrm{msec}$. Attempts to improve performance with the beat pattern at short durations, by increasing the speed of movement, simply make matters worse. This is consistent with the findings of Derrington and Badcock (1985) that the temporal resolution of the mechanism analysing the motion of beats is poorer than that of the mechanism which analyses the motion of either high spatial-frequency or low spatial-frequency luminance patterns.

It is important to consider exactly what kind of difference between first-order and second-order motiondetecting mechanisms is implied by the fact that secondorder mechanisms require the stimulus to be of a longer duration, and what other results might be predicted from this observation. Perhaps the most plausible explanation 

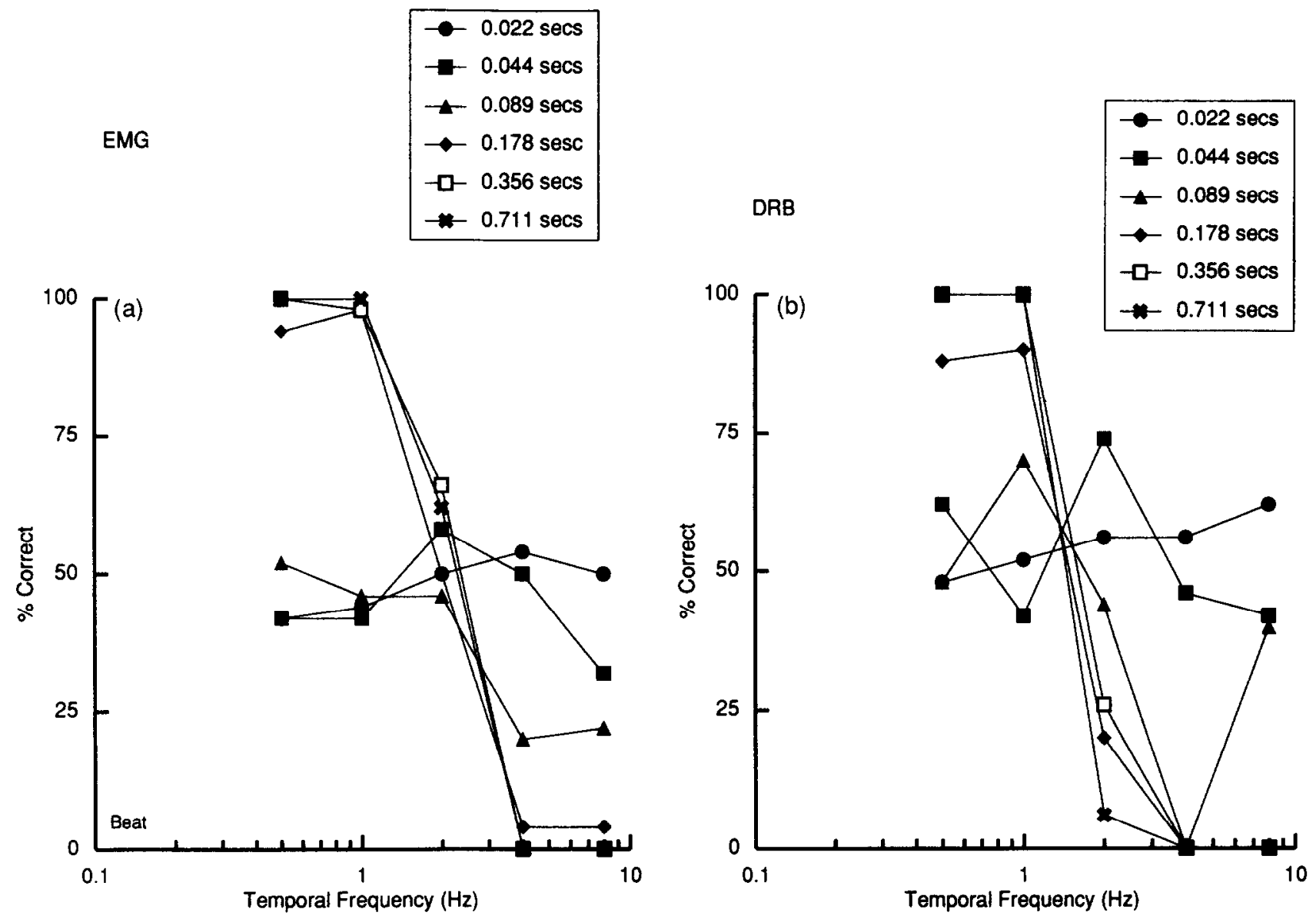

FIGURE 6. Data from Fig. 5(e,f) replotted as functions of temporal frequency with duration as parameter.

for the difference would be that second-order motion analysis mechanisms are subject to more severe low-pass temporal filtering than are first-order motion analysis mechanisms. This increased low-pass filtering may be necessary to allow the second-order mechanisms to correlate over long delays without aliasing. We are indebted to an anonymous referee for the suggestion that discriminations based on second-order mechanisms might also show longer latencies.

One question that arises from this work is whether the time it takes to analyse the motion of the beat pattern is limited by the mechanism which analyses the motion, as we have tacitly assumed, or by the mechanism which analyses its spatial structure, since this must obviously precede the analysis of its motion (Cavanagh \& Mather, 1989). The reports of our observers indicate quite unambiguously that the problem is in the analysis of motion, as even at the shortest duration the spatial structure of the beat patterns is quite obvious.

A related question is that of whether the poor performance with beat patterns depends on contrast. We chose to use stimuli of fixed physical contrast, and it is likely that the beat patterns were closer to their threshold than the other patterns we used. However, as stated above, the beat patterns were supra-threshold, and the fact that they may have been closer to threshold than were the other patterns used is unlikely to have caused the observed deficits in performance. Indeed the avail- able evidence suggests that supra-threshold increases in contrast may actually impair direction-discrimination performance (Boulton, 1987; Derrington \& Goddard, 1989).

Recent work in this laboratory shows that the difference we report here between first-order and second-order motion is equally clear when the contrast of each pattern is normalized with respect to its detection threshold (Cropper, 1992; Cropper \& Derrington, 1990, 1993).

\section{REFERENCES}

Adelson, E. H. \& Bergen, J. R. (1985). Spatiotemporal energy models for the perception of motion. Journal of the Optical Society of America A, 2, 284-299.

Adelson, E. H. \& Movshon, J. A. (1982). Phenomenal coherence of moving visual patterns. Nature, 300, 523-525.

Anstis, S. M. (1970). Phi movement as a subtraction process. Vision Research, 10, 1411-1430.

Anstis, S. M. (1980). The perception of apparent movement. Philosophical Transactions of the Royal Solciety of London B, 290, 153-168.

Boulton, J. C. (1987). Two mechanisms for the detection of slow motion. Journal of the Optical Society of America A, 4, 1634-1642.

Braddick, O. (1974). A short-range process in apparent motion. Vision Research, 14, 519-527.

Braddick, O. J. (1980). Low-level and high-level processes in apparent motion. Philosophical Transactions of the Royal Society of London $B, 290,137-151$.

Cavanagh, P. \& Mather, G. (1989). Motion: The long and short of it. Spatial Vision, 4, 103-129. 
Chandler, J. P. (1965). STEPIT, Quantum Chemistry Program Exchange, Bloomington, Ind.

Chubb, C. \& Sperling, G. (1989). Two motion perception mechanisms revealed through distance driven reversal of apparent motion Proceedings of the National Academy of Sciences, 86, 2985-2989.

Cropper, S. J. (1992). Motion discrimination: Different patterns, differ ent detectors. Ph.D. thesis. University of Newcastle upon Tyne, England.

Cropper, S. J. \& Derrington, A. M. (1990). The effects of contrast and duration on the discrimination of direction of motion in beats and chromatic gratings. Perception, 19, 353.

Cropper, S. J. \& Derrington, A. M. (1993). Motion of chromatic stimuli: First-order or second-order? Vision Research. Submitted.

Derrington, A. M. (1987). Distortion products in geniculate X cells: A physiological basis for masking by spatially modulated gratings? Vision Research, 27, 1377-1386.

Derrington, A. M. (1990). Mechanisms for coding luminance patterns: Are they really linear? In Blakemore, C. (Ed.), Vision, coding and efficiency (pp. 175-184). Cambridge: Cambridge University Press.

Derrington, A. M. \& Badcock, D. R. (1985). Separate detectors for simple and complex grating patterns? Vision Research, 25 , 1869-1878.

Derrington, A. M. \& Badcock, D. R. (1992). Two-stage analysis of the motion of 2-dimensional patterns, what is the first stage? Vision Research, 32, 691-698.

Derrington, A. M. \& Goddard, P. A. (1989). Failure of motion discrimination at high contrasts: Evidence for saturation. Vision Research, 29, 1767-1776.

Derrington, A. M. \& Suero, M. (1991). Motion of complex patterns is computed from the perceived motions of their components. Vision Research, 31, 139-149.

Derrington, A. M., Badcock, D. R. \& Holroyd, S. A. (1992). Analysis of the motion of 2-dimensional patterns: Evidence for a secondorder process. Vision Research, 32, 699-707.

Henning, G. B. (1988). Spatial-frequency tuning as a function of temporal frequency and stimulus motion. Journal of the Optical Society of America A, 5, 1362-1373.
Henning, G. B., Hertz, B. G. \& Broadbent, D. E. (1975). Some experiments bearing on the hypothesis that the visual system analyses spatial patterns in independent bands of spatial. Vision Research, $15,887-897$.

Morgan, M. J. \& Cleary, R. (1992). Ambiguous motion in a two-frame sequence. Vision Research, 32, 2195-2198.

Pelli, D. G. \& Zhang, L. (1991). Accurate control of contrast on microcomputer displays. Vision Research, 31, 1337-1350.

Petersik, J. T. \& Pantle, A. (1979). Factors controlling the competing sensations produced by a bistable stroboscopic motion display. Vision Research, 19, 143 154.

Reichardt, W. (1961). Autocorrelation, a principle for the evaluation of sensory information by the central nervous system. In Rosenblith, W. A. (Ed.), Sensory communication (pp. 303-317). New York: Wiley.

Robson, J. G. (1966). Spatial and temporal contrast sensitivity functions of the visual system. Journal of the Optical Society of America, 56, 1141-1142.

van Santen, J. P. H. \& Sperling, G. (1984). Temporal covariance model of human motion pcrception. Journal of the Optical Society of America A, 1, 451-473.

Ulman, S. (1979). The interpretation of visual motion. Cambridge, Mass.: MIT Press.

Watson, A. B. \& Ahumada. A. J. J. (1985). Model of human visual-motion sensing. Journal of the Optical Society of America A, 2. $322-342$.

Wilson, H. R., Ferrera, V. P. \& Yo, C. (1992). A psychophysically motivated model for two-dimensional motion perception. Visual Neuroscience, 9. 79-97.

Yo, C. \& Wilson, H. R. (1992). Perceived direction of moving two-dimensional patterns depends on duration, contrast and eccentricity. Vision Research, 32, 135-147.

Acknowledgements - This research was supported by SERC grants GR/G 00730 and GR/G 07982. D. R. Badcock was on sabbatical leave from the University of Melbourne. 